\title{
Estimación gravimétrica del grosor cortical en el Golfo de California
}

\author{
Gravimetric crustal thickness estimation in the Gulf of California
}

Anaid Fragoso-Irineo ${ }^{1, *}$, Javier Lazcano ${ }^{1}$, Roberto S. Molina-Garza ${ }^{2}$, Alexander Iriondo ${ }^{2,3}$

${ }^{1}$ Posgrado en Ciencias de la Tierra, Centro de Geociencias, Universidad Nacional Autónoma de México, Juriquilla, Querétaro, México.

${ }^{2}$ Centro de Geociencias, Universidad Nacional Autónoma de México, Blvd. Juriquilla no. 3001,76230, Querétaro, México.

${ }^{3}$ Department of Geosciences, University of Arizona, 1040 E. 4th Street, Tucson, Arizona 85721

* Autor para correspondencia:

(A. Fragoso-Irineo)afragoso@geociencias.unam.mx

\begin{abstract}
Cómo citar este artículo:
Fragoso-Irineo, A., Lazcano, J., MolinaGarza, R.S., Iriondo, A., 2021, Estimación gravimétrica del grosor cortical en el Golfo de California: Boletín de la Sociedad Geológica Mexicana, 73 (1), A270820. http://dx.doi. org/10.18268/BSGM2021v73nla270820
\end{abstract}

Manuscrito recibido: 5 de Febrero de 2020 Manuscrito corregido: 10 de Agosto de 2020 Manuscrito aceptado: 24 de Agosto de 2020

La revisión por pares es responsabilidad de la Universidad Nacional Autónoma de México.

\section{RESUMEN}

En este estudio se implementa la inversión 3D de datos gravimétricos satelitales mediante el programa 3DINVER.M para modelar el grosor de la corteza en el rift del Golfo de California. Este rift oblicuo separa dextralmente la península de Baja California del continente de Norteamérica desde el Mioceno, periodo en el que se produjo el cambio desde un régimen tectónico fundamentalmente extensional al transtensional presente. A lo largo de su eje, el rift del Golfo de California se encuentra en una fase de oceanización incipiente en varias cuencas de su mitad meridional, mientras que al norte la presencia de corteza oceánica es controvertida. A consecuencia de esta particularidad, la corteza de la cuenca marina de Golfo y la de regiones terrestres adyacentes presentan importantes diferencias de densidad y grosor, siendo ambos parámetros fundamentales del método empleado, y, por ello, se realiza un modelo específico para cada dominio. Los modelos de grosor cortical generados se compararon con las estimaciones puntuales previas basadas en métodos sísmicos, obteniéndose que el $98 \%$ de los puntos confrontados del modelo marino y el $87 \%$ del terrestre se ajustan a un rango de $\pm 6 \mathrm{~km}$ de diferencia, con diferencias promedio absolutas de $1.5 \mathrm{~km}$ y de $3.5 \mathrm{~km}$ respectivamente. La cuenca del Golfo en los modelos evoluciona a lo largo de su eje y desde el sur al norte desde una corteza híper-adelgazada $(\sim 8-12 \mathrm{~km})$, característica de corteza oceánica a transicional, hasta una corteza continental moderadamente adelgazada $(\sim 12-18 \mathrm{~km})$. En al área extendida circundante, que completa la denominada como Provincia Extensional del Golfo, la corteza continental varía habitualmente entre $\sim 15 \mathrm{y} \sim 28 \mathrm{~km}$, mientras que en sus flancos la corteza llega a alcanzar los 35-40 $\mathrm{km}$ en los dominios más engrosados (Sierra Madre Occidental y Meseta del Colorado). Asimismo, con base en las estimaciones del grosor cortical y la geología regional, se propone una nueva demarcación para la Provincia Extensional del Golfo.

Palabras clave: grosor cortical, Moho, gravimetría, inversión 3D, Golfo de California, rift.

\section{ABSTRACT}

In this study $3 D$ inversion of satellite gravimetric data with 3DINVER.M program is applied to model the depth of the crust in the Gulf of California rift. This rift dextrally separates Baja California Peninsula from the North American continent since Miocene, period in which the change occurred from a fundamentally extensional tectonic regime to the present transtensional. Incipient oceanization occurs in various southern sectors along the rift axis, while to the north the presence of oceanic crust is controversial. As a consequence, the crust of the Gulf marine basin and that of adjacent terrestrial regions exhibit important differences in density and thickness, both fundamental parameters of the method, and therefore, we opted to model independently each domain. The crustal thickness models were compared with previous punctual estimates based on seismic methods, obtaining that $98 \%$ of the confronted points of the marine model and $87 \%$ of the terrestrial model are adjusted to a range of \pm $6 \mathrm{~km}$ difference, with mean differences of $1.5 \mathrm{~km}$ and $3.5 \mathrm{~km}$, respectively. In our models, along the Gulf basin axis and from the south to the north evolves from a hyper-thinned crust $(\sim 8-12 \mathrm{~km})$, characteristic of oceanic to transitional crust, to a moderately thinned continental crust $\sim \sim 12-18$ $\mathrm{km})$. In the surrounding extended area, known as the Gulf Extensional Province, the continental crust usually varies between $\sim 15$ and $\sim 28 \mathrm{~km}$, while on this province flanks the crust reaches -35-40 km in the thickened domains (Sierra Madre Occidental and Colorado Plateau). Based on our estimates of crustal thickness and the regional geology, a new demarcation for the Gulf Extensional Province is proposed.

Keyzwords: crustal thickness, Moho, gravimetry, 3D inversion, Gulf of California, rift. 


\section{Introducción}

La estructura cortical en el Golfo de California ha sido modificada enormemente desde que inició la actual etapa de ruptura continental, un proceso cuyo mayor efecto en la corteza es un severo adelgazamiento diferencial. El grosor cortical en un territorio, o lo que es lo mismo, la profundidad a la que se encuentra la discontinuidad de Mohorovičić (Moho), está condicionado por múltiples fenómenos geodinámicos (localización y tipo de deformación, magmatismo, sedimentación, etc.), y, por lo tanto, se trata de un parámetro fundamental en la interpretación tectónica. En el rift del Golfo de California el conocimiento del grosor cortical proviene casi exclusivamente de estimaciones puntuales en redes o a lo largo de transectos a partir de diversos métodos geofísicos (e.g. Thatcher y Brune, 1973; Sánchez-Zamora, 1981; Couch et al., 1991; Lewis et al., 2001; Reyes et al., 2001; González-Fernández et al., 2005; Lizarralde et al., 2007; López-Pineda et al., 2007; Persaud et al., 2007; Bashir et al., 2011; Gaite et al., 2015; Fernández y Pérez-Campos, 2017; Espíndola et al., 2017; Paulssen y de Vos, 2017).

Si bien los cálculos del Moho con métodos sísmicos pueden tener una gran precisión $(\sim 1-2$ $\mathrm{km})$, los métodos gravimétricos tienen la ventaja de disponer de una mayor densidad de datos y, en cambio, ofrecen una mayor cobertura (Chen y Tenzer, 2017; Ebadi et al., 2019; Zhao et al., 2020); siendo por lo tanto especialmente útiles en la elaboración de modelos regionales o de gran escala.

En este estudio se ofrece una estimación del grosor cortical en toda el área del rift del Golfo de California, mediante la inversión gravimétrica 3D de la anomalía completa de Bouguer, obtenida a partir de datos gravimétricos satelitales de libre acceso (Global Gravimetry Model 05C de Ries et al., 2016). El procedimiento de inversión gravimétrica se realiza con el programa 3DINVER.M, diseñado por Gómez-Ortiz y Agarwal (2005), que ya ha sido puesto a prueba satisfactoriamente en otros escenarios regionales (e.g. Block et al., 2009;
Tugume et al., 2013; Van der Meijde et al., 2013; O'Donnell et al., 2014). 3DINVER.M se basa en el método de Parker-Oldenburg, que permite modelar la profundidad de la superficie del Moho a partir de una profundidad de referencia y el contraste de densidad promedio estimado para el límite corteza-manto. Siendo estos dos parámetros en promedio muy diferentes en la corteza híbrida del núcleo del rift y en la puramente continental de sus márgenes, se opta por generar dos modelos, marino y terrestre, que se adaptan bien a cada dominio. Ambos se han puesto a prueba con los resultados de estudios geofísicos previos, obteniéndose diferencias mínimas $( \pm 6 \mathrm{~km})$ en la mayoría de los casos.

A partir de los modelos generados, se han podido modificar los límites de la Provincia Extensional del Golfo, propuestos por otros autores (Figura 1; Stock y Hodges, 1989; Calmus et al., 2010). Los nuevos límites se ajustaron de acuerdo con cambios significativos en el espesor de la corteza y están respaldados por otros criterios geológicos, especialmente por la localización de estructuras distensivas mayores (e.g. complejos de núcleo metamórfico de Sonora).

\section{Contexto geológico}

El Golfo de California es un rift oblicuo continental con una depresión central (mar de Cortés) compartimentada en una serie de subcuencas de tipo pull-apart, alrededor de la cual se desarrolla de forma asimétrica una amplia área extendida (Lonsdale, 1989) (Figura 1). Este rift forma parte de un margen transformante más extenso que continúa al norte por el sistema de fallas de San Andrés, hasta enlazar finalmente con la zona de fractura de Mendocino, en el borde sur de la placa Gorda.

El rifting en el Golfo de California es un proceso continuo en cuya evolución se distinguen dos grandes fases, atribuibles a las etapas en modo ancho y estrecho típicas de los rifts. La primera inicia con la extensión esencialmente ortogonal 
asociada a la provincia sur del Basin and Range (Henry y Aranda-Gómez, 2000; Ferrari et al., 2002), etapa en la que se forma la gran provincia ígnea oligo-miocénica de la Sierra Madre Occidental (Aguirre-Díaz y Labarthe-Hernández, 2003), que actuó en la siguiente etapa como una barrera ante la deformación. La segunda etapa, de carácter transtensivo dextral y que propiamente se conoce como el rift del Golfo de California, surgió hace ca. 12.5 Ma, cuando la dorsal del Pacífico Oriental alcanzó la trinchera de forma oblicua, provocando el cese de la subducción de la placa Farallón y el acoplamiento entre la placa del Pacífico y la de Norteamérica (Atwater y Stock,
1998). Desde entonces, el movimiento hacia el norte de la placa del Pacífico respecto a la de Norteamérica ocasionó el desgarre de la península de Baja California, cuyo desplazamiento se ha estimado en la parte norte del Golfo en 300-350 km (Oskin et al., 2001).

La cuenca marina del Golfo puede dividirse en dos sectores, norte y sur, a partir de la latitud $\mathrm{N} 28^{\circ}$, aproximadamente. En el sector meridional se genera nueva corteza oceánica en dorsales, como la de Alarcón desde hace tan sólo 2-3 Ma (Lizarralde et al., 2007); mientras que en el sector septentrional, la oceanización no se ha alcanzado (e.g. Persaud et al., 2007; González-Fernández et al.,

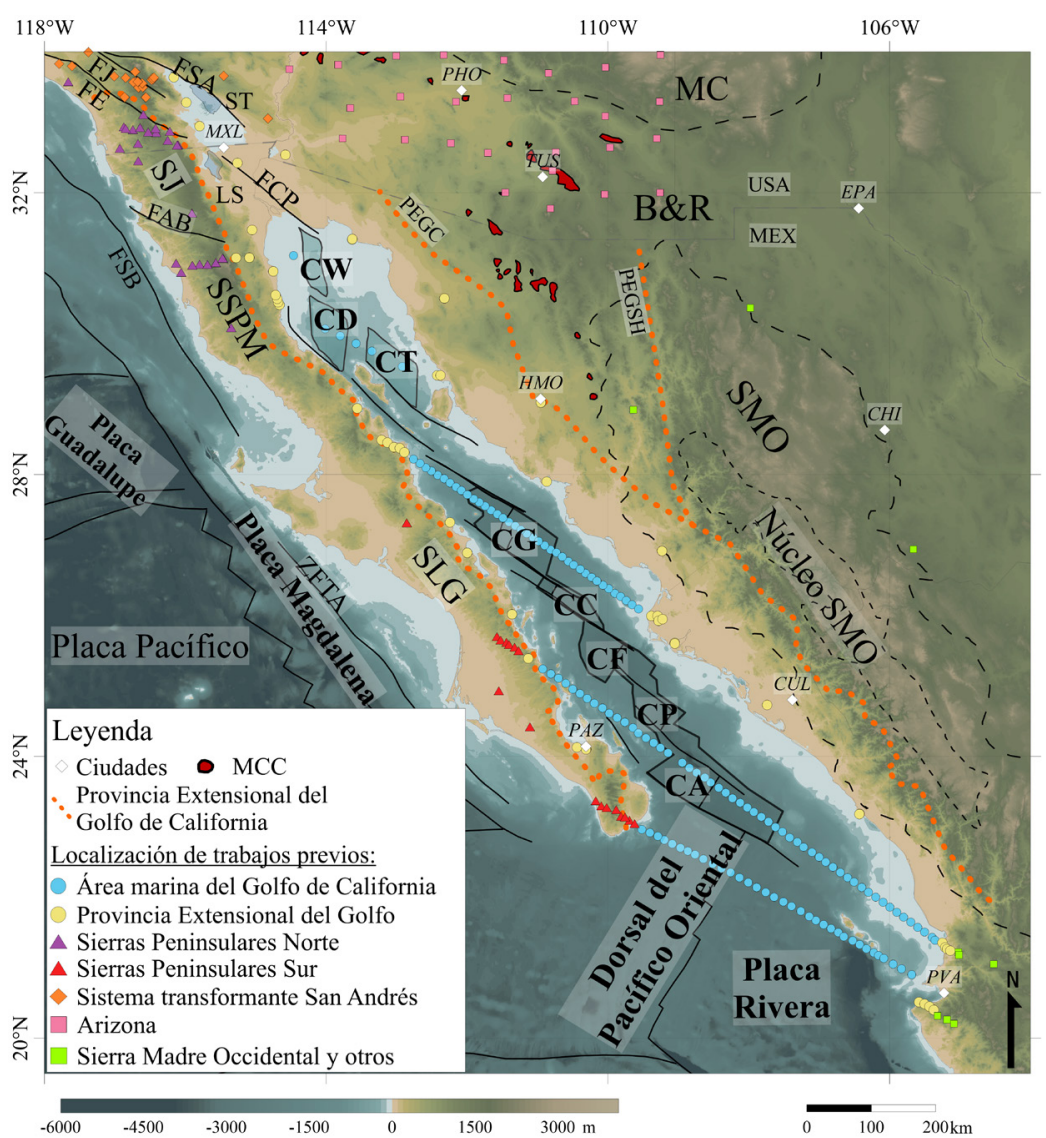

Figura 1 Mapa del área de estudio con la localización de las estaciones de los trabajos previos. Ciudades: CHI, Chihuahua; CUL, Culiacán; EPA, El Paso; HMO, Hermosillo; MXL, Mexicali; PAZ, La Paz; PHO, Phoenix; PVA, Puerto Vallarta; TUS, Tucson. Fallas: FAB, Falla Agua Blanca; FCP, Falla Cerro Prieto; FE, Falla Elsinore; FJ, Falla San Jacinto; FSA, Falla de San Andrés; FSB, Falla San Benito; ZFTA, zona de falla ToscoAbreojos. Cuencas: $C A$, Cuenca Alarcón; $C C$, Cuenca Carmen; $C D$, Cuenca Delfín; $C F$, Cuenca Farallón; $C G$, Cuenca Guaymas; $C P$, Cuenca Pescadero; CT, Cuenca Tiburón; CW, Cuenca Wagner. Regiones: B\&R, Basin \& Range; LS, Laguna Salada; MC, Meseta del Colorado; SJ, Sierra Juárez; SLG, Sierra La Giganta; SMO; Sierra Madre Occidental; SSPM, Sierra San Pedro Mártir; ST, depresión Salton. Límites de la Provincia Extensional del Golfo: PEGC, Calmus et al. (2010); PEGSH, Stock y Hodges (1989). Complejos de núcleo metamórficos tomados de Spencer (2018). Modelo de relieve GMTR 3.6. (Ryan et al. 2009). 
2005) o sería incipiente (Wang et al., 2009; MartínBarajas et al., 2013). El área circundante extendida durante la fase en modo estrecho del rift completa la Provincia Extensional del Golfo (Karig y Jensky, 1972; Moore, 1973; Gastil et al., 1975), que de acuerdo con Stock y Hodges (1989) limita al oeste con el Escarpe del Golfo y al este con la Sierra Madre Occidental; mientras que al norte lo hace con el sistema de fallas de San Andrés y la provincia del Basin and Range, y al sur desaparece bajo el Cinturón Volcánico Transmexicano.

\section{Trabajos previos}

Las estimaciones de la profundidad del Moho en el Golfo de California en estudios previos se realizaron empleando diversos métodos geofísicos, fundamentalmente a partir de datos de sísmica de refracción, funciones receptor y gravimetría. Exceptuando los modelos globales CRUST (Bassin et al., 2000; Laske et al., 2013) y un modelo de inversión 3D de datos gravimétricos isostáticos en la mitad norte del Golfo (García-Abdeslem, 2017), las mediciones son puntuales, habitualmente a lo largo de perfiles o en redes de varias estaciones (Figura 1). De forma congruente con la geodinámica, los trabajos previos están de acuerdo en que el grosor cortical se adelgaza notablemente desde los flancos (Península de Baja California y Sierra Madre Occidental) hacia el núcleo del rift (mar de Cortés o Golfo de California s.s.).

En la cuenca marina del Golfo de California, los estudios previos coinciden en que el grosor cortical es menor en el sector meridional. En el sur, la profundidad del Moho estimada varía habitualmente entre $\sim 8.5$ y $10 \mathrm{~km}$ (Thatcher y Brune, 1973; Sánchez-Zamora, 1981; Couch et al., 1991; Lizarralde et al., 2007); mientras que en el norte alcanza los $~ 15-16 \mathrm{~km}$ (Lewis et al., 2001; González-Fernández et al., 2005), o incluso hasta $\sim 20 \mathrm{~km}$ de acuerdo con Thatcher y Brune (1973).

En la franja extendida circundante a la cuenca del Golfo de California, se ha estimado que el grosor cortical varía entre $\sim 15$ y $25 \mathrm{~km}$ (SánchezZamora, 1981; Couch et al., 1991; Lewis et al.,
2001; Reyes et al., 2001; González-Fernández et al., 2005; Persaud et al., 2007; Fernández y PérezCampos, 2017). Hacia el interior del continente, ya en la provincia del Basin and Range de Sonora y Arizona, la profundidad del Moho aumenta significativamente hasta los $\sim 22-32 \mathrm{~km}$ (Couch et al., 1991; Espíndola et al., 2017; Fernández y PérezCampos, 2017; García-Abdeslem, 2017; Paulssen y de Vos, 2017). Al norte del área de estudio, en el segmento transformante que conecta el Golfo de California con el sistema de fallas de San Andrés, el grosor cortical presenta variaciones importantes y se han calculado profundidades de entre $\sim 21$ y $\sim 34$ km (Ichinose et al., 1996; Lewis et al., 2000; Zhu y Kanamori, 2000).

En cuanto a las áreas que delimitan el rift y que, no han sido significativamente extendidas el grosor cortical, se incrementa hasta superar los $30 \mathrm{~km}$ en la Sierra Madre Occidental ( 40 km, SánchezZamora, 1981; $\sim 50$ km, Bonner y Herrin, 1999; $\sim 32-40 \mathrm{~km}, \sim 30 \mathrm{~km}$, Gaite et al., 2015; Espíndola et al., 2017; 42 km, García-Abdeslem, 2017) y en la Meseta de Colorado ( 40-45 km, Bashir et al., 2011); mientras que en las Sierras Peninsulares de California y Baja California las estimaciones son también típicas de corteza continental pero muy variables ( 38-42 km, Lewis et al., 2001; 32-42 km, Reyes et al., 2001; 42 km, López-Pineda et al., 2007; 28-37 km, Persaud et al., 2007; 32 km, Espíndola et al., 2017; 28-37 km, Fernández y Pérez-Campos, 2017; 38-42 km, GarcíaAbdeslem, 2017; 28.5-36 km, Paulssen y de Vos, 2017).

\section{Métodos}

\subsection{MÉTODO DE INVERSIÓN}

Los modelos de la profundidad del Moho se generaron mediante el programa 3DINVER.m para Matlab (Gómez-Ortiz y Agarwal, 2005). 3DINVER.m se fundamenta en el método de Parker-Oldenburg, que permite calcular la geometría y posición de cierta interfase de densidad en una malla de datos gravimétricos. Parker (1972) concibió un algoritmo para calcular 
la atracción gravitacional ejercida por una capa bidimensional de densidad constante, a partir de una serie infinita de trasformadas de Fourier del relieve de la capa. La anomalía gravimétrica de la capa se expresa de la siguiente forma:

$F(\Delta g)=-2 \pi G \rho e^{\left(-|\vec{k}| z_{0}\right)} \sum_{n=1}^{k^{n-1}} \frac{|\vec{k}|^{n-1}}{n !} F\left[h(\vec{x})^{n}\right]$

donde $F$ es la transformada de Fourier de la anomalía gravimétrica, $G$ es la constante universal gravitacional, $p$ es el contraste de densidad a través de la interfase, $k$ es el número de onda, $h(x)$ es la profundidad del relieve y $z_{0}$ es la profundidad de referencia (promedio) de la interfase horizontal.

Oldenburg (1974) modificó el algoritmo de Parker (1972) para producir el relieve de la capa mediante un procedimiento de inversión iterativo a partir de la anomalía gravimétrica, y lo formuló de la siguiente manera:

$$
F[\Delta g]=-\frac{F[\Delta g] e^{|\vec{k}| z_{0}}}{2 \pi G \rho}-\sum_{n=2}^{\infty} \frac{|\vec{k}|^{n-1}}{n !} F\left[(h(\vec{x}))^{n}\right]
$$

donde $p$ es el contraste de densidad entre los dos medios y los demás parámetros son equivalentes (ecuación 1). Este nuevo algoritmo es capaz de procesar eficazmente gran cantidad de datos, manteniendo sus propiedades numéricas de estabilidad y una adecuada convergencia con la ayuda de un filtro pasa bajas (high-cut filter, $H C F$ ). El filtro pasa-bajas se constriñe de acuerdo a lo siguiente:

$$
\begin{array}{cc}
H C F(k)=\frac{1}{2}\left[1+\cos \left(\frac{k-2 \pi W H}{2(S H-W H)}\right)\right] & \text { para } W H<k<S H \\
H C F(k)=0 & \text { cuando } k>S H \\
H C F(k)=1 & \text { cuando } k<W H
\end{array}
$$

donde la frecuencia $k$ se puede interpretar también como $1 / \lambda$, siendo $\lambda$ la longitud de onda en kilómetros; $W H$ es el corte de filtro de frecuencias bajas (smaller cut-off frequency) y SH es el corte de

\begin{tabular}{|c|c|c|c|c|}
\hline $\begin{array}{l}\text { Prof. } \\
\text { Ref. } \\
(\mathrm{km})\end{array}$ & $\begin{array}{l}\text { Contraste } \\
\text { de densidad } \\
\left(\mathrm{kg} / \mathrm{m}^{3}\right)\end{array}$ & RMS & $\begin{array}{l}\text { Prof. } \\
\text { Min. } \\
(\mathrm{km})\end{array}$ & $\begin{array}{l}\text { Prof. } \\
\text { Max. } \\
(\mathrm{km})\end{array}$ \\
\hline 10 & 200 & 0.0225 & -24 & 47 \\
\hline 10 & 250 & 0.0112 & -16 & 41 \\
\hline 10 & 500 & 0.0055 & -3 & 26 \\
\hline 10 & 600 & 0.0032 & -1 & 23 \\
\hline 15 & 200 & 0.031 & -21 & 53 \\
\hline 15 & 250 & 0.0134 & -13 & 47 \\
\hline 15 & 270 & 0.0087 & -11 & 44 \\
\hline 15 & 300 & 0.0142 & -8 & 42 \\
\hline 15 & 400 & 0.0124 & -2 & 35 \\
\hline 15 & 450 & 0.0086 & 0 & 33 \\
\hline 15 & 500 & 0.0062 & 1 & 32 \\
\hline 15 & 600 & 0.0035 & 4 & 29 \\
\hline 15 & 700 & 0.0022 & 6 & 27 \\
\hline 15 & 800 & 0.0014 & 7 & 25 \\
\hline 17.5 & 200 & 0.0364 & -20 & 56 \\
\hline 17.5 & 250 & 0.0148 & -11 & 50 \\
\hline 17.5 & 270 & 0.0095 & -9 & 47 \\
\hline 17.5 & 300 & 0.0153 & -6 & 44 \\
\hline 17.5 & 400 & 0.0132 & 0 & 38 \\
\hline 17.5 & 450 & 0.0091 & 2 & 36 \\
\hline 17.5 & 500 & 0.0065 & 4 & 34 \\
\hline 17.5 & 600 & 0.0037 & 6 & 32 \\
\hline 20 & 200 & 0.043 & -18 & 59 \\
\hline 20 & 250 & 0.0163 & -10 & 52 \\
\hline 20 & 270 & 0.0108 & -7 & 50 \\
\hline 20 & 300 & 0.0166 & -4 & 47 \\
\hline 20 & 350 & 0.0087 & 0 & 44 \\
\hline 20 & 400 & 0.014 & 1 & 41 \\
\hline 20 & 450 & 0.0096 & 4 & 39 \\
\hline 20 & 500 & 0.0069 & 6 & 37 \\
\hline 20 & 600 & 0.0039 & 8 & 34 \\
\hline 25 & 200 & 0.0666 & -15 & 64 \\
\hline 25 & 300 & 0.0198 & 0 & 53 \\
\hline 25 & 600 & 0.0044 & 13 & 40 \\
\hline 30 & 200 & 0.0956 & -11 & 70 \\
\hline 30 & 300 & 0.089 & -4 & 59 \\
\hline 30 & 400 & 0.0182 & 11 & 52 \\
\hline 30 & 600 & 0.005 & 18 & 45 \\
\hline
\end{tabular}
filtro de frecuencias altas (greater cut-off frequency), que están representados por $1 / \lambda \mathrm{km}$.
Tabla 1. Ensayos realizados con los parámetros de profundidad de referencia y contraste de densidad. En negrita los valores correspondientes a los modelos generados (marino y terrestre). 
De esta forma, para calcular la profundidad del Moho, equivalente a la interfase densidad de las ecuaciones previas, 3DINVER.m requiere que se asignen los valores de los parámetros de profundidad de referencia $\left(z_{0}\right)$ y contraste de densidad $(p)$, así como un criterio de convergencia y los valores de filtros de frecuencia $W H$ y $S H$ para disminuir el error (RMS). El código calcula la profundidad del Moho mediante un proceso iterativo en el que se aplica la transformada inversa de Fourier hasta alcanzar un RMS menor al criterio de convergencia asignado, o al llegar a 10 iteraciones por defecto. Gómez-Ortiz y Agarwal (2005) sugieren que los valores del criterio de convergencia y filtros de frecuencia $\mathrm{WH}$ y $\mathrm{SH}$ sean elegidos por prueba y error, y que se definan en función de la precisión esperada.

Como ya se mencionó anteriormente, la existencia de corteza oceánica/transicional en el Mar de Cortés, en contraposición a la corteza continental del resto del área emergida, imposibilita cuantificar conjuntamente los parámetros $z_{0}$ y $p$ en ambos dominios, por lo que se realizaron dos modelos independientes. Considerando los rangos de los datos publicados, se elaboraron los modelos variando los valores de $z_{0}$ cada $5 \mathrm{~km}$ entre los $10 \mathrm{~km}$ y $30 \mathrm{~km}$ de profundidad, y los de $p$ en intervalos de $50 \mathrm{~kg} / \mathrm{m}^{3}$ entre los 200 y $800 \mathrm{~kg} / \mathrm{m}^{3}$; fijándose a su vez el filtro pasa bajos, $S H$ con filtro de frecuencia de 1/100 km y $W H$ de $0 \mathrm{~km}$ (Tabla 1; Figura 3). En ambos casos, el modelo preliminar elegido fue aquel con mejor equilibrio entre un menor RMS y una mayor concordancia con las profundidades del Moho recopiladas. Finalmente, los modelos definitivos se obtuvieron tras ajustar el filtro pasabajos siguiendo el criterio anterior. Se variaron los filtros de frecuencia cada $1 / 100 \mathrm{~km}$ desde $1 / 100$ $\mathrm{km}$ a $1 / 1,000 \mathrm{~km}$, y cada $1 / 500 \mathrm{~km}$ hasta $1 / 2,500$ $\mathrm{km}$, cuidando de que el RMS arrojado no fuese mayor a uno.

\subsection{DATOS GRAVIMÉTRICOS}

En la elaboración de los modelos de espesor cortical se utilizó el modelo estático de gravedad GRACE Gravimetry Model 05C (GGM05C; Ries et al., 2016), cuya resolución es de 2 minutos de arco $(\sim 3.66$ km). El GGM05C resulta de la combinación de los modelos gravimétricos satelitales GRACE (01/2003 a 05/2013; Ries et al., 2016) y GOCE

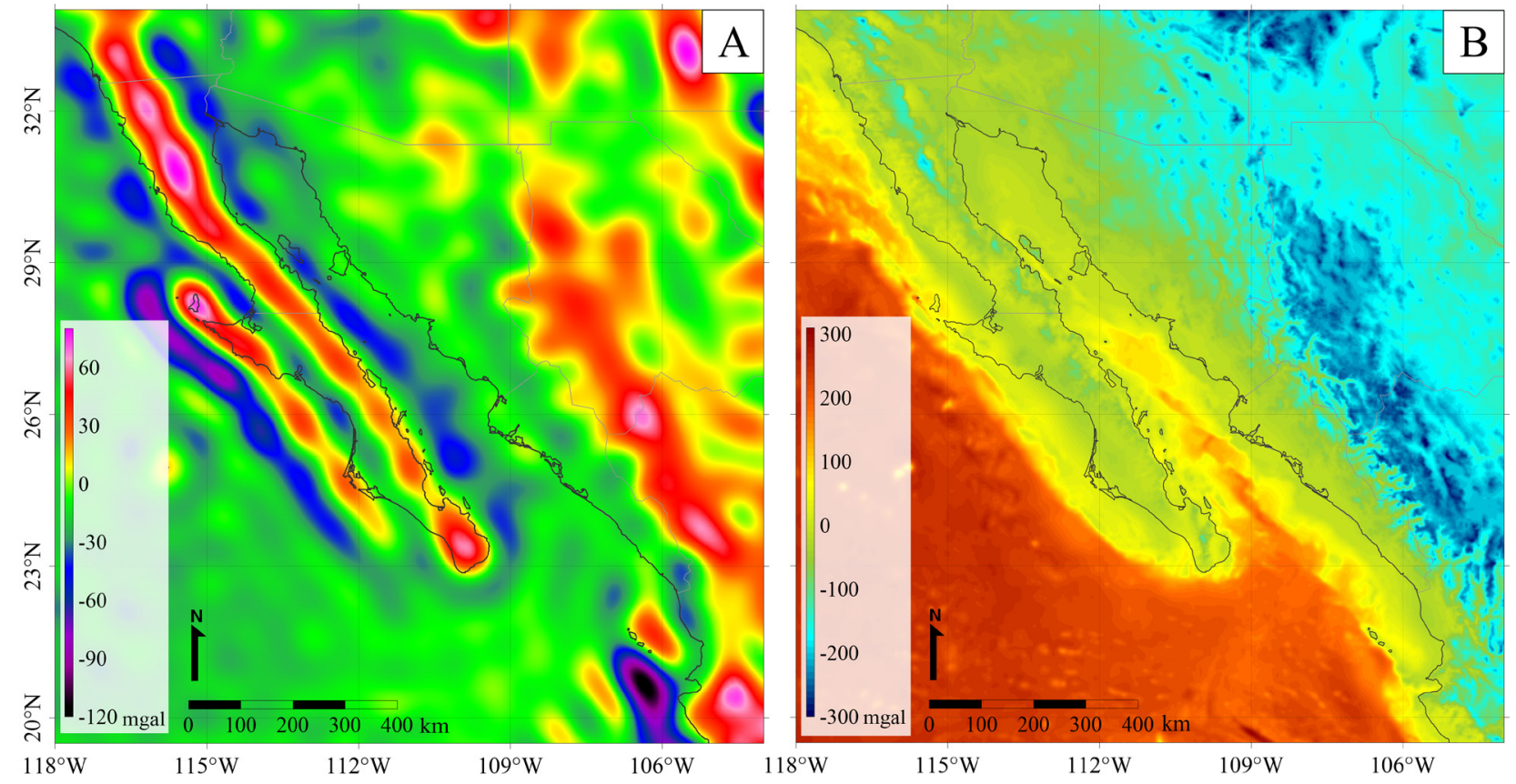

Figura 2 (A) Mapa de anomalía de aire libre del modelo GGM05C (Ries et al., 2016), descargado de http://icgem.gfz-potsdam.de/home (Barthelmes y Köhler, 2016; Ince et al., 2019). (B) Mapa de anomalía de Bouguer completa generada para este estudio. 
(11/2009 a 10/2013; Gruber y Rummel, 2013), y el modelo de anomalía gravimétrica superficial DTU13 (Andersen et al., 2014). Se empleó una malla de datos gravimétricos de 950×950 nodos con un espaciado de $3.4 \times 3.6 \mathrm{~km}$ en el área comprendida entre las latitudes $44^{\circ} \mathrm{N}$ y $14^{\circ} \mathrm{N}$ y las longitudes $126^{\circ} \mathrm{W}$ y $96^{\circ} \mathrm{W}$, superior a las dimensiones del área de estudio $\left(34-19.5^{\circ} \mathrm{N}\right.$; $118-104^{\circ} \mathrm{W}$ ), a fin de evitar problemas de borde. Los datos se descargaron en forma de anomalía de aire libre de la página http://icgem.gfz-potsdam. de/home (Barthelmes y Köhler, 2016; Ince et al., 2019) (Figura 2A).

Puesto que el programa 3DINVER.M opera con la anomalía de Bouguer completa (ABC) (Figura 2B), a los datos gravimétricos de entrada se les aplicaron las correcciones de Bouguer, de la curvatura de Bullard y la topográfica, empleando para ello el modelo de elevación GMTR versión 3.6 (Ryan et al., 2009), cuya resolución en tierra es de 30 m en México y 10 m en Estados Unidos, y en el mar desciende hasta los $100 \mathrm{~m}$ (descargado de https://www.gmrt.org/GMRTMapTool/).

Siguiendo la ecuación para la determinación de ABC de Lowrie (2007) se calcularon las correcciones de Bouguer normal (continente) y de Bouguer de mar, tomando los valores de densidad convencionales de $2.67 \mathrm{~g} / \mathrm{cm}^{3}$ para la corteza y de $1.03 \mathrm{~g} / \mathrm{cm}^{3}$ para el agua marina. La corrección de la gravedad por la curvatura de la Tierra, o de Bullard, se realizó de acuerdo con Lee y Acharya (2017), quienes emplean una aproximación a partir de Whitman (1991); y como sugieren estos autores, se utilizaron un radio terrestre de $6,730 \mathrm{~km}$
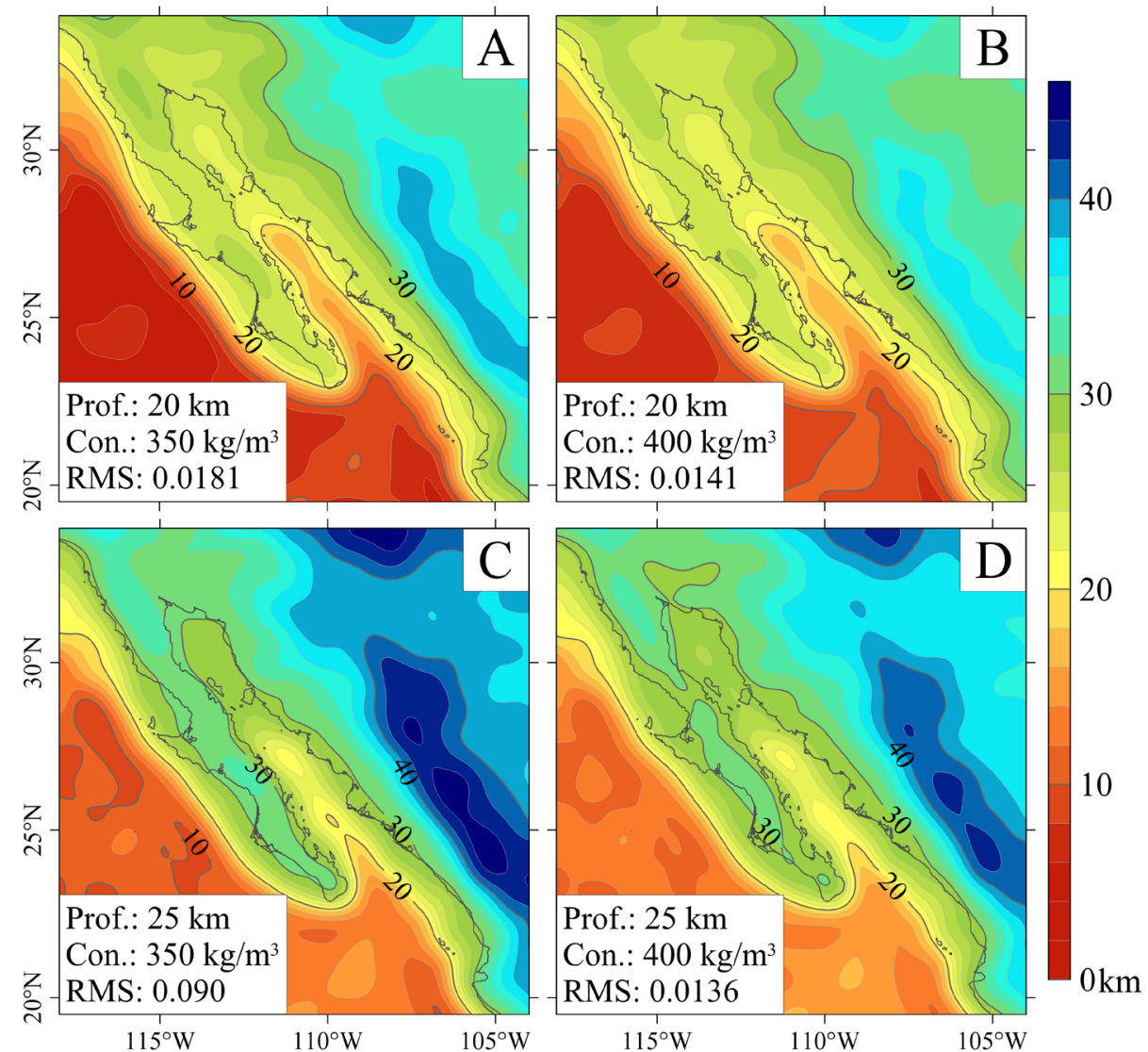

Figura 3 (A) Modelo terrestre definitivo generado con una profundidad de referencia de $20 \mathrm{~km}$ y un contraste de densidad de $350 \mathrm{~kg} /$ $\mathrm{m}^{3}$. Los restantes modelos muestran los efectos de la modificación de estos parámetros principales. (B) El aumento del contraste de densidad a $400 \mathrm{~kg} / \mathrm{m}^{3}$ repercute en un menor grosor cortical al previsto en las regiones menos extendidas o engrosadas (e.g. Sierra Madre Occidental). (C) El aumento de la profundidad de referencia a $25 \mathrm{~km}$ resultó en grosores corticales excesivamente elevados, alcanzándose los $\sim 30-35 \mathrm{~km}$ en la franja costera que bordea la cuenca del Golfo, donde habitualmente se reportan valores de $\sim 20-25$ $\mathrm{km}$. (D) Modelo en el que se modificaron ambos parámetros y el grosor cortical resultante es significativamente mayor al esperado. 
y una anchura de $166.735 \mathrm{~km}$ (radio de Bullard), que coincide con el límite externo indicado en el sistema de Hayford-Bowie (Hayford y Bowie, 1912).

Finalmente, el cálculo de la corrección topográfica se realizó con el módulo Gravity Terrain del paquete Oasis Montaj de Geosoft (2015), el cual usa una combinación de los métodos descritos por Kane (1962) y Nagy (1966). Se mantuvieron los valores generados por defecto por el módulo (distancia externa: $300 \mathrm{~km}$; distancia interna: $3.4 \times 3.6 \mathrm{~km}$ ), que los calcula a partir del tamaño de la malla ingresada.

\section{Resultados}

\subsection{MODELOS DE GROSOR GORTICAL}

Se han realizado dos modelos del grosor cortical en el Golfo de California, para la porción marina y la terrestre (Figura 4). En el modelo marino, los valores preferidos fueron $15 \mathrm{~km}$ para la profundidad de referencia, $600 \mathrm{~kg} / \mathrm{m}^{3}$ para el contraste de densidad, y 1/100 km para $S H$ y
$1 / 250 \mathrm{~km}$ para $W H$ del filtro pasa-bajos $(\mathrm{RMS}=$ 0.0131). En el modelo terrestre, se optó por $20 \mathrm{~km}$ de profundidad de referencia, $350 \mathrm{~kg} /$ $\mathrm{m}^{3}$ de contraste de densidad, y 1/100 km para $S H$ y $1 / 300 \mathrm{~km}$ para $W H$ del filtro pasa-bajos $(\mathrm{RMS}=0.0184)$. Finalmente, ambos modelos se combinaron a partir de la línea de costa (Figura 5).

Cabe señalar que en el modelo terrestre la profundidad de referencia es sorprendentemente baja, más cuando toda la superficie terrestre presenta un mayor grosor cortical. Sin embargo, otros valores aparentemente más realistas derivan en modelos que no se ajustan adecuadamente a los datos de los trabajos previos y por ello se descartaron. En el caso del contraste de densidad, con base en las relaciones empíricas de Barton (1986), entre velocidades sísmicas y la densidad de la corteza atravesada, los valores escogidos se ciñen a los rangos calculados a partir de los datos publicados. De esta forma, las velocidades promedio en el ámbito terrestre para la corteza inferior varían entre $6.4-7.0 \mathrm{~km} / \mathrm{s}$, y para el manto entre $7.58-7.9 \mathrm{~km} / \mathrm{s}$ (Hendricks y Plescia, 1991), siendo equiparables a densidades de 2,580-3,210 $\mathrm{kg} / \mathrm{m}^{3}$ y $3,100-3,400 \mathrm{~kg} / \mathrm{m}^{3}$ respectivamente, de

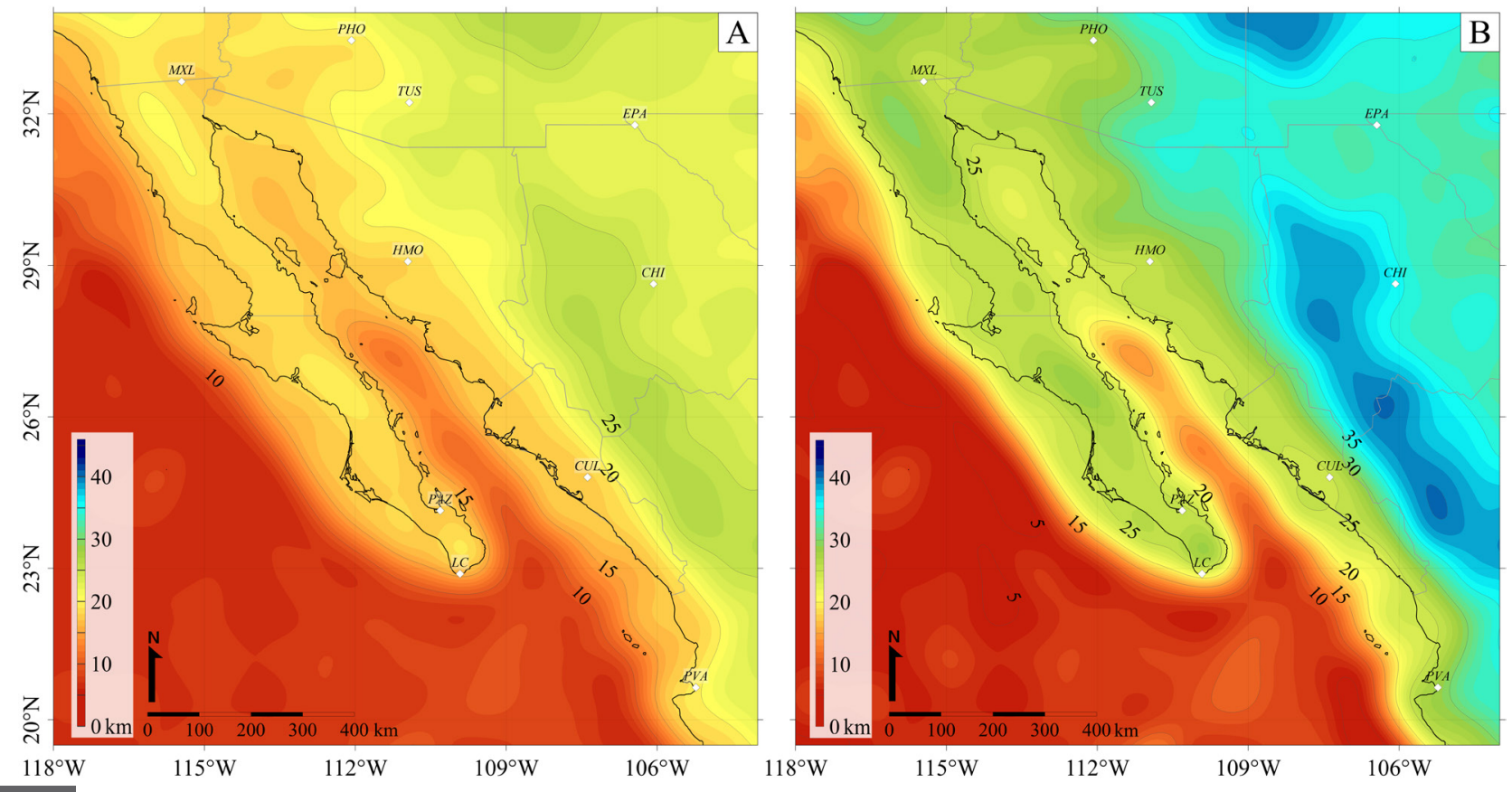

Figura 4 Modelos del grosor cortical en el Golfo de California generados: (A) marino y (B) terrestre. 
lo que se obtiene un valor promedio del contraste de densidad de $385 \mathrm{~kg} / \mathrm{m}^{3}$, casi idéntico al empleado, y máximo de $820 \mathrm{~kg} / \mathrm{m}^{3}$.

En el ámbito marino las velocidades promedio para la corteza inferior son de $6.8 \mathrm{~km} / \mathrm{s}$ y para el manto de $7.9 \mathrm{~km} / \mathrm{s}$ (González-Fernández et al., 2005; Lizarralde et al., 2007), comparables con densidades de $2,690-3,160 \mathrm{~kg} / \mathrm{m}^{3}$ y $3,130-3,400$ $\mathrm{kg} / \mathrm{m}^{3}$ respectivamente, y que supone un contraste de densidad promedio de $340 \mathrm{~kg} / \mathrm{m}^{3}$ y máximo de $710 \mathrm{~kg} / \mathrm{m}^{3}$, entre los que se sitúa el valor escogido.

Las anomalías gravimétricas calculadas para cada modelo, junto con su diferencia con la ABC de entrada, se representan en la figura 6. En ambos mapas se observa una mayor diferencia en aquellas zonas con cambios pronunciados en la pendiente de la ABC (Figura 2B), como ocurre en la Sierra Madre Occidental, en la península de Baja California, en el límite de la plataforma continental del Pacífico o en la parte sur del Golfo de California, que pudiera estar asociada a altas frecuencias en la $\mathrm{ABC}$, relacionadas con fuentes superficiales. No obstante, las diferencias generalmente oscilan tan solo entre -25 a +25 mgal en toda el área de estudio (color blanco, Figura 6C y 6D).

\subsubsection{MODELO MARINO}

En el sector septentrional del Golfo de California, el grosor cortical es sistemáticamente mayor que en el meridional (Figura 4A). Los valores de profundidad del Moho obtenidos en nuestro modelo son similares a los presentados por Lewis et al. (2001) y González-Fernández et al. (2005),

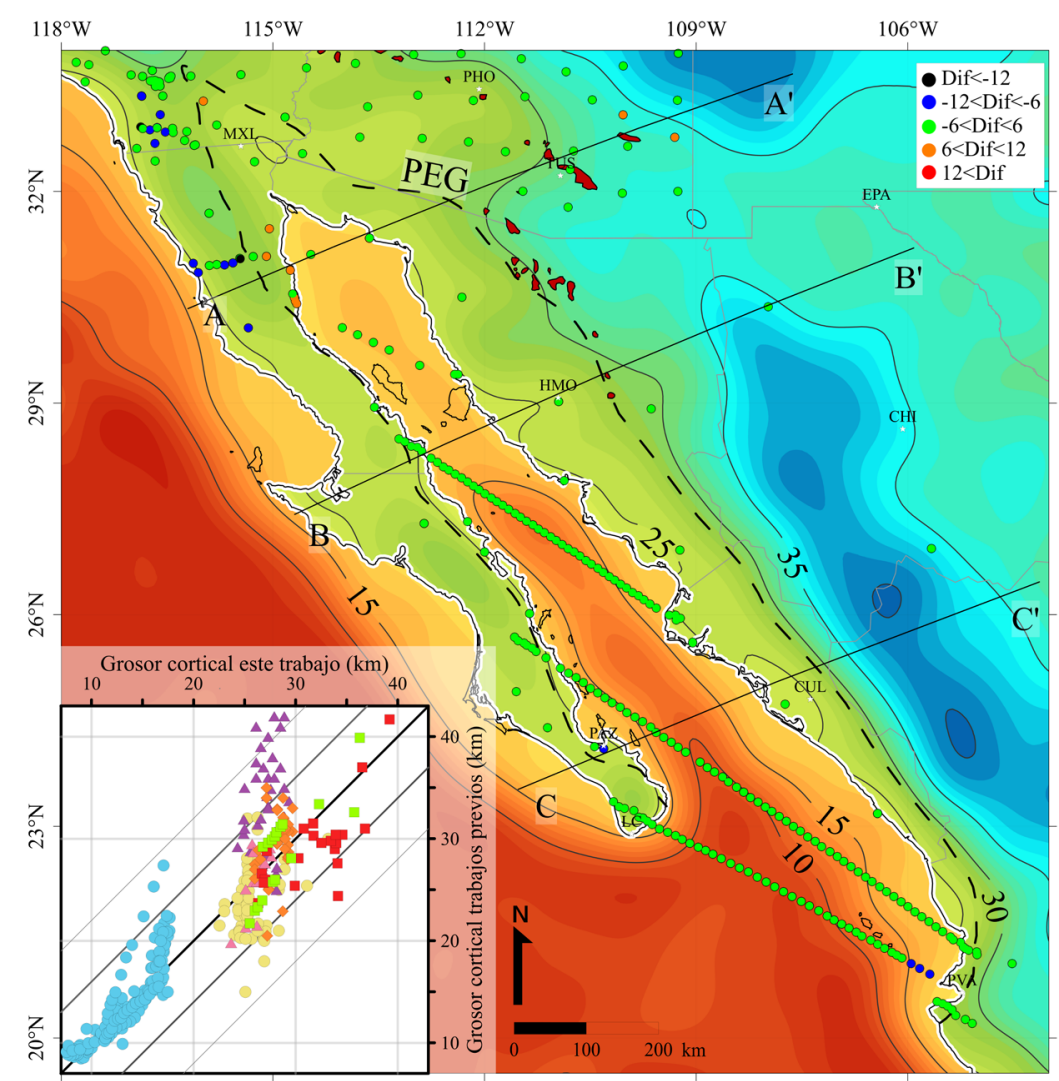

Figura 5 Modelo del grosor cortical compuesto con las estaciones de los trabajos previos clasificadas según su diferencia respecto a nuestro estudio y la localización de los perfiles realizados. PEG: Provincia Extensional del Golfo propuesta en este estudio a partir de los mapas de grosor cortical y la geología regional. La línea blanca sobre la costa indica el solapamiento entre el modelo marino y el terrestre. Polígonos rojos: complejos de núcleo metamórficos tomados de Spencer (2018). Esquina inferior izquierda: gráfica del grosor cortical en los trabajos previos frente a los de este estudio; los símbolos aluden a la ubicación de los trabajos previos (ver Figura 1 ). Las líneas paralelas marcan la diferencia en la profundidad del Moho (línea central 0 km; líneas internas \pm 6 km; líneas externas \pm 12 km). 
con una diferencia de $\pm 2 \mathrm{~km}$ en la mayoría de los puntos contrastables. Estas profundidades oscilan entre los $16.5 \mathrm{~km}$ en la parte noreste de la cuenca Delfin Norte y entre 17 y $18 \mathrm{~km}$ fuera del eje del rift.

Los grosores corticales más bajos del sector meridional coinciden con la sucesión de cuencas de tipo pull-apart de Guaymas, Farallón, Pescadero y finalmente más al sur Alarcón, donde el grosor cortical alcanza los 11.8, 11.3, 9.6 y $7.7 \mathrm{~km}$ respectivamente. Los valores de la profundidad del Moho de nuestro modelo, comparados con los obtenidos por Lizarralde et al. (2007) en sus perfiles sísmicos, son prácticamente idénticos $(<1$ $\mathrm{km}$ ) fuera de la franja litoral, en la que sí llegan a existir diferencias notables de entre 2 y $10 \mathrm{~km}$.

\subsubsection{MODELO TERRESTRE}

La Provincia Extensional del Golfo presenta grosores corticales de 22 a $27 \mathrm{~km}$, pudiendo establecer un promedio de $25 \mathrm{~km}$. Comparado con las profundidades del Moho puntuales publicadas, la diferencia absoluta promedio es de $3.16 \mathrm{~km}$ (Figura 4B). En la planicie costera de Sinaloa $(\sim 25$ $\mathrm{km})$, la corteza se engrosa bruscamente desde las estribaciones de la Sierra Madre Occidental hasta su núcleo $(\sim 40 \mathrm{~km})$, y más al este se vuelve a adelgazar, aunque en menor medida a lo observado en el margen oriental del Golfo (Figura 7; perfil La Paz-Culiacán). Nuestro modelo en esta región es congruente con las aportadas en la mayoría de los trabajos previos (Couch et al., 1991; Kerdan, 1992; Campos-Enríquez et al., 1994).

$\mathrm{Al}$ norte de Hermosillo, y coincidiendo aproximadamente con la divisoria entre el sector norte y sur de la cuenca del Golfo, la corteza está adelgazada de forma más heterogénea (Figura 7; perfil Hermosillo). El espesor cortical característico de $\sim 25 \mathrm{~km}$ al sur se prolonga hasta el río Gila en Arizona de forma irregular (Figura
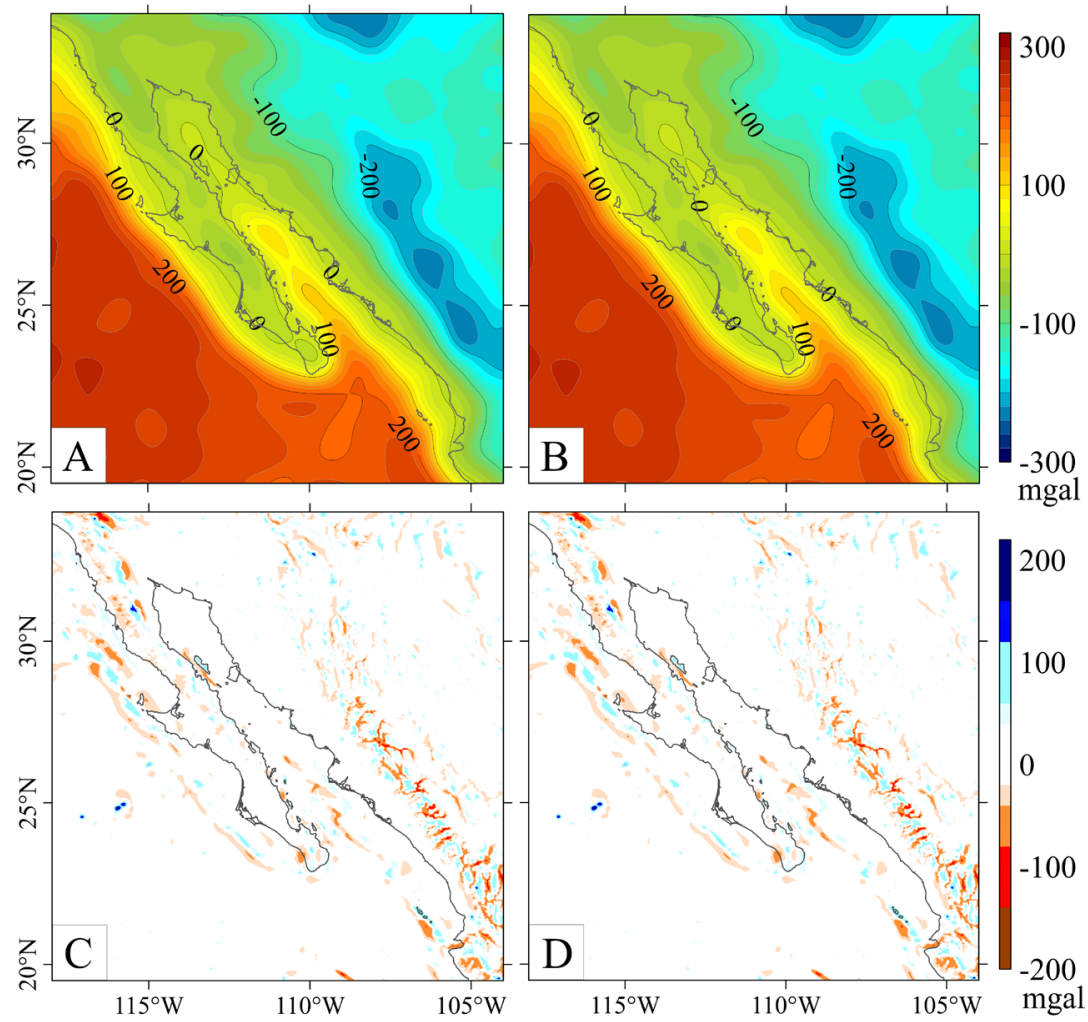

Figura 6 Anomalías gravimétricas calculadas para cada uno de los modelos con su diferencia con la anomalía completa de Bouguer. A) Mapa de anomalía gravimétrica calculada para el modelo marino. B) Mapa de anomalía gravimétrica calculada para el modelo terrestre. C) Diferencia entre los mapas de anomalías gravimétricas para el modelo marino. D) Diferencia entre los mapas de anomalías gravimétricas para el modelo terrestre. 

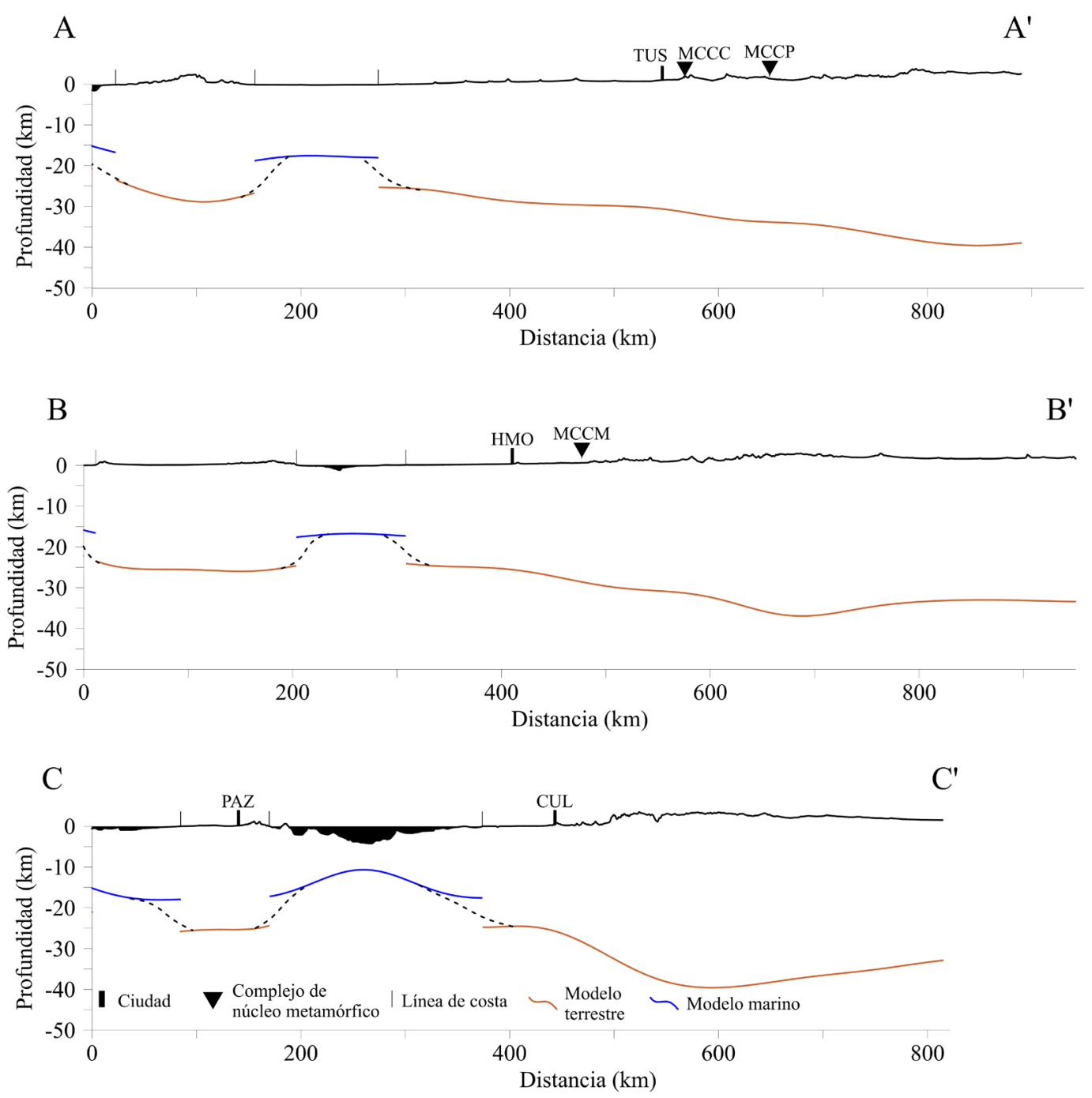

Figura 7 Perfiles topografía vs. grosor cortical. (A) Perfil de Tucson; (B) perfil de Hermosillo; (C) perfil de La Paz-Culiacán. Véase la discusión en el texto. Ciudades: CUL, Culiacán; HMO, Hermosillo; PAZ, La Paz; TUS, Tucson. Complejos de núcleo metamórficos: MCCC, Santa Catalina; MCCM, Mazatán; MCCP, Pinaleño.

7; perfil Tucson), interrumpido por dos franjas significativamente menos adelgazadas ( 26-28 $\mathrm{km})$ y fuertemente oblicuas al eje rift. Estas franjas se sitúan sobre las latitudes $30^{\circ} \mathrm{N}$ y $32^{\circ} \mathrm{N}$ y hacia el este se engrosan gradualmente juntándose finalmente en una región poco adelgazada $(\leq 35$ $\mathrm{km})$ entre la Sierra Madre Occidental ( 30-40 $\mathrm{km})$ y la Meseta de Colorado ( 35-40 km).

$\mathrm{Al}$ norte el Golfo de California se prolonga hasta la depresión Salton con un grosor cortical sobre el eje del rift de $\sim 25 \mathrm{~km}$. En cambio, más al noroeste en las Sierras Transversales (Traverse Ranges) predomina la deformación transpresiva asociada a la curvatura del margen transformante
(Sylvester y Smith, 1976) y la corteza tiene un grosor normal de $\sim 29-31 \mathrm{~km}$. No obstante, superpuesta a la traza de la falla Elsinore, se observa una franja adelgazada $(\sim 27 \mathrm{~km})$.

El flanco occidental del rift presenta un grosor cortical muy heterogéneo, con profundidades máximas de $\sim 30 \mathrm{~km}$ y mínimas de $\sim 25 \mathrm{~km}$. Los mayores espesores se dan en la parte norte en las sierras Juárez (29.5 km) y San Pedro Mártir (28.8 km), que están separadas por la falla Agua Blanca (e.g. Allen et al., 1960; Dixon et al., 2002), y al sur bajo la sierra La Giganta $(28.8 \mathrm{~km})$ y en el bloque de Los Cabos (28 km). En la sierra La Giganta aflora extensamente el grupo volcánico oligo-miocénico 
Comondú, sincrónico con el volcanismo mioceno de la Sierra Madre Occidental (e.g. Umhoefer et al., 2001; Duque-Trujillo et al., 2015). El bloque Los Cabos se trata de un fragmento de basamento batolítico exhumado durante el Neógeno (Fletcher et al., 2000), probablemente emparentado con el Batolito de Puerto Vallarta (Schaaf et al., 2000), que a su vez podría estarlo con el Batolito de las Sierras Peninsulares (Schaaf et al., 2019).

\subsection{VALIDAGIÓN}

La validez de nuestros modelos se ha puesto a prueba mediante la comparación con los datos de función receptor, sísmica de reflexión y refracción, publicados en trabajos previos (Figura 5). En total fueron 320 datos de 191 estaciones terrestres y 129 marinas las que se emplearon en esta tarea (Figura 1). De igual forma que en los trabajos de Van der Meijde et al. (2013) y Tugume et al. (2013) se filtraron los datos de sísmica de acuerdo con un error menor o igual a $6 \mathrm{~km}$ (cuando figura el valor de la incertidumbre) y en el caso de los datos de función receptor se desdeñaron aquellos que no tuvieran más de 10 sismos como referencia. Bajo este criterio, se obtuvo que el porcentaje de similitud con los datos previos en el modelo marino es del 98\% y en el modelo terrestre del $87 \%$, cifras muy altas que consideramos que validan ambos modelos. Estos porcentajes de similitud se traducen en diferencias promedio absolutas de $1.5 \mathrm{~km}$ en el modelo marino y de $3.5 \mathrm{~km}$ en el terrestre.

Para facilitar la visualización de este procedimiento de validación, se graficaron nuestros resultados frente a los datos de referencia de los trabajos previos, agrupados en diferentes regiones (Figura 5). Las menores discrepancias se registran en el área marina del Golfo y en el extremo sur de la Península de Baja California, con diferencias absolutas promedio de $1.5 \mathrm{~km}$ y $1.43 \mathrm{~km}$ respetivamente. Tienen una buena correlación los puntos localizados en el sistema transformante de San Andrés $(2.5 \mathrm{~km})$, en la Sierra Madre Occidental $(2.7 \mathrm{~km})$, en la Provincia Extensional del Golfo de California (3 km) y en el suroeste de Arizona $(3 \mathrm{~km})$. Por el contrario, la discrepancia es alta en las Sierras Peninsulares del norte, donde la diferencia promedio absoluta asciende hasta $6.7 \mathrm{~km}$, superando así el límite que consideramos aceptable. Esta diferencia la atribuimos a que los valores de los parámetros involucrados en la elaboración del modelo no representan adecuadamente la gruesa y densa corteza inferior máfica que compone la porción occidental de la península (Langenheim et al., 2014; Fragoso-Irineo, 2014).

\section{Discusión}

En nuestro modelo marino se reafirma la diferencia del grosor cortical entre los sectores septentrional y meridional de la cuenca del Golfo puesta de manifiesto en todos los trabajos previos (Figura 4A). Las profundidades del Moho obtenidas en el sector meridional a lo largo del eje del rift son propias de una corteza continental hiperextendida, en el sentido de Reston (2009). En la mitad sur de este sector, los valores son compatibles con la existencia de corteza oceánica en amplias regiones; mientras que en su mitad norte la corteza oceánica estaría confinada en ciertas cuencas de segundo orden, como ya han señalado otros estudios previamente (e.g. Lizarralde et al., 2007). En cambio, en la parte septentrional del Golfo, las profundidades promedio superiores a $15 \mathrm{~km}$ del sector norte corresponden a un tipo de corteza continental adelgazada moderadamente, que aún dista de alcanzar la etapa transicional registrada al sur, salvo de forma muy localizada en cualquier caso (Wang et al., 2009; Martín-Barajas et al., 2013).

El modelo terrestre muestra por norma general una relación directa positiva entre hipsometría y grosor cortical. Esta circunstancia es evidente por ejemplo en la Sierra Madre Occidental y en la Meseta de Colorado (Figura 7; perfiles La PazCuliacán y Tucson), y denota que gran parte del territorio está en aparente equilibrio isostático. Puntualmente, hay zonas que no cumplen estrictamente esta relación, así en la sierra Juárez de Baja California la profundidad del Moho 
es mayor que en la aledaña sierra de San Pedro Mártir, aun dándose las mayores altitudes en esta última. En este caso, el diferente grosor cortical posiblemente tiene su origen en particularidades de los arcos mesozoicos que componen ambas sierras, Santiago Peak al norte en sierra Juárez y Alisitos en la sierra de San Pedro Mártir (Wetmore et al., 2002; 2003).

Las regiones con un mayor grosor cortical corresponden a las menos extendidas o bien a las que habiendo sufrido un proceso distensivo temprano registraron luego un voluminoso magmatismo. En el primer caso destacan la Meseta de Colorado y el Batolito de las Sierras Peninsulares; en el segundo, la Sierra Madre Occidental y la sierra La Giganta. El elevado grosor cortical demostrado por los datos geofísicos bajo el núcleo de la Sierra Madre Occidental, el cual no está afectado significativamente por extensión post-miocénica (e.g. Ferrari et al., 2013), no se trata de un rasgo heredado, es consecuencia de la formación de esta gran provincia ígnea o LIP en su acrónimo anglosajón (Bryan, 2007; Sheth, 2007). Se ha propuesto la existencia de una gruesa raíz batolítica compuesta por rocas máficas a intermedias feldespáticas (Cameron y Robinson, 1990) o la profusión de intrusiones de magmas máficos en su corteza inferior (Ferrari et al., 2007). De forma similar a lo que ocurre en la Sierra Madre Occidental el engrosamiento bajo el coetáneo grupo Comondú (e.g. Umhoefer et al., 2001; Duque-Trujillo et al., 2015) en la sierra La Giganta puede estar ligado a las raíces plutónicas del volcanismo subaéreo.

En Sonora, el espesor cortical es heterogéneo, reflejo de una evolución geológica compleja. El grado de extensión sufrido en este territorio es, a su vez, muy variable, siendo máximo en los complejos de núcleo metamórficos (Nourse et al., 1994; Vega-Granillo y Calmus, 2003; Wong y Gans, 2003, 2008; Wong et al., 2010). La región más adelgazada se encuentra limitada al este por estos complejos de núcleo metamórficos (Figura 7; perfiles Hermosillo y Tucson), dispuestos en una franja que continúa al norte en Arizona y que forma parte de un extenso cinturón a lo largo del margen Cordillerano hasta el sur de Canadá (e.g. Coney, 1980; Armstrong, 1982). Los complejos de núcleo metamórfico en el área de estudio se encuentran sobre corteza continental que varía entre 26 y $33 \mathrm{~km}$ de profundidad, y que previamente a la extensión cenozoica podría haber sido engrosada durante la orogenia Larámide, cuya arquitectura habría condicionado la distribución de los complejos (Coney y Harms, 1984).

El modelo terrestre ha permitido trazar un nuevo perímetro de la Provincia Extensional del Golfo (Figura 4B), que modifica los de Stock y Hodges (1989) y Calmus et al. (2010). Entendiendo esta provincia como el segmento meridional activo del margen transformante del suroeste de Norteamérica, proponemos que su borde occidental se sitúa aproximadamente sobre el Escarpe del Golfo desde Los Cabos hasta la depresión Salton, y que el borde oriental linda al sur con la Sierra Madre Occidental, se solapa al norte con el cinturón de complejos de núcleo metamórficos de Sonora y casi a la latitud $32^{\circ}$ $\mathrm{N}$, vira hacia el oeste para confluir finalmente en la depresión Salton. El espesor cortical en la Provincia Extensional del Golfo oscila entre $~ 25$ y $\sim 27 \mathrm{~km}$, salvo en una pequeña área al noroeste de Hermosillo en la latitud $30^{\circ} \mathrm{N}$. Coincide que en esta región el basamento está constituido por el complejo paleoproterozoico Bámuri, emparentado con la provincia cortical Mojave del paleocontinente de Laurencia (Castiñeiras et al., 2004; Farmer et al., 2005; Iriondo y Premo, 2011). Consideramos por lo tanto que la anomalía se debe a la existencia de un bloque más rígido, que habría condicionado el grado de extensión en esa parte del Golfo (Lazcano-Prieto, 2018).

\section{Conclusiones}

En este estudio se presentan dos modelos del grosor cortical en el Golfo de California, para el ámbito marino y el terrestre, realizados a partir de datos gravimétricos satelitales del modelo GGM05C, con el programa 3DINVER.M para 
Matlab (Gómez-Ortiz y Agarwal, 2005). Para garantizar la confiabilidad de ambos modelos, se cotejaron las profundidades del Moho obtenidas con los datos puntuales previamente publicados. En la validación se admitió un valor máximo de $\pm 6 \mathrm{~km}$ de diferencia, obteniéndose que el 98\% de los puntos confrontados del modelo marino y el $87 \%$ del terrestre se ajustan a este rango, cifras que a nuestro juicio abalan su validez.

En el modelo marino para la cuenca del Golfo contrasta el grosor cortical en el sector meridional, que oscila entre $\sim 8 \mathrm{y} \sim 15 \mathrm{~km}$, con el del sector septentrional menos adelgazado, cuya mínima profundidad del Moho es de $\sim 16.5 \mathrm{~km}$. Estas profundidades son compatibles con corteza de tipo oceánica a transicional a lo largo del eje del rift en el sur y continental moderadamente adelgazada en el norte. En el modelo terrestre para la región emergida circundante, se han distinguido varios dominios morfotectónicos con importantes cambios en el grosor cortical, lo que ha permitido redibujar los límites de la Provincia Extensional del Golfo. El espesor cortical en esta región extendida varía entre $\sim 25$ y $\sim 27$ km, y sólo puntualmente supera los $30 \mathrm{~km}$. En los flancos del rift, las profundidades del Moho se sitúan entre $\sim 28 \mathrm{~km}$ (occidental) y $\sim 40 \mathrm{~km}$ (oriental).

En conclusión, este estudio demuestra la posibilidad de obtener modelos del grosor cortical en amplias regiones geológicamente complejas, a partir de datos gravimétricos satelitales mediante procesamientos de inversión 3D. Para ello, es necesario elaborar diferentes modelos adaptados a los tipos de corteza presentes, continental pura y transicional, debido a que el procedimiento requiere cuantificar una serie de parámetros dependientes de la composición y estructura de la corteza involucrada.

\section{Agradecimientos}

El presente trabajo se enmarca en los estudios para optar al grado de Doctora de Anaid Fragoso Irineo, quien agradece al CONACyT la beca que le fue otorgada. Apreciamos los comentarios y sugerencias del revisor David Escobedo Zenil (Universidad Nacional Autónoma de México) y otro revisor anónimo, de David Gómez Ortiz (Universidad Rey Juan Carlos), creador del programa 3DINVER.M, y de Juan Manuel Espinosa Cardeña, que mejoraron sustancialmente el manuscrito; de la misma forma, agradecemos la disposición del editor en jefe Antoni Camprubí (Universidad Nacional Autónoma de México).

\section{Referencias}

Aguirre-Díaz, G.J., Labarthe-Hernández, G., 2003, Fissure ignimbrites: Fissure-source origin for voluminous ignimbrites of the Sierra Madre Occidental and its relationship with Basin and Range faulting: Geology, 31(9), 773-776. https://doi.org/10.1130/ g19665.1

Allen, G.R., Silver, L.T., Stehli, F.G., 1960, Agua Blanca fault - a major transverse structure of northern Baja California, Mexico: Geological Society of America Bulletin, 71(4), 467-482. https://doi. org/10.1130/0016-7606(1960)71 [467:abfmt $\mathrm{s}] 2.0 . \mathrm{co} ; 2$

Andersen, O.B., Knudsen, P., Kenyon, S.C., Holmes, S., 2014, Global and arctic marine gravity field from recent satellite altimetry (DTU13), in 76th EAGE Conference and Exhibition 2014, 1-5. http://doi. org/10.3997/2214-4609.20140897

Armstrong, R.L., 1982, Cordilleran metamorphic core complexes-From Arizona to southern Canada: Annual Review of Earth and Planetary Sciences, 10(1), 129-154. https:// doi.org/10.1146/annurev. ea.10.050182.001021

Atwater, T., Stock, J., 1998, Pacific-North America plate tectonics of the Neogene southwestern United States: an update: International Geology Review, 40(5), 375-402. https:// doi.org/10.1080/00206819809465216

Barthelmes, F., Köhler, W., 2016, International Centre for Global Earth Models (ICGEM), in: Drewes, H., Kuglitsch, F., Adám, J. et al., 
The Geodesists Handbook 2016, Journal of Geodesy, 90 (10), 907-1205. http://doi. org/10.1007/s00190-016-0948-z

Barton, P.J., 1986, The relationship between seismic velocity and density in the continental crust - a useful constraint?: Geophysical Journal International, 87(1), 195-208. https://doi.org/10.1111/j.1365-246x.1986. tb04553.x

Bashir, L., Gao, S.S., Liu, K.H., Mickus, K., 2011, Crustal structure and evolution beneath the Colorado Plateau and the southern Basin and Range Province: Results from receiver function and gravity studies: Geochemistry, Geophysics, Geosystems, 12(6), Q06008. http://doi.org/10.1029/2011GC003563

Bassin, C., Laske, G., Masters, G., 2000, The current limits of resolution for surface wave tomography in North America: EOS Transactions American Geophysical Union, 81, F897.

Block, A.E., Bell, R.E., Studinger, M., 2009, Antarctic crustal thickness from satellite gravity: Implications for the Transantarctic and Gamburtsev Subglacial Mountains: Earth and Planetary Science Letters, 288(12), 194-203. https://doi.org/10.1016/j. epsl.2009.09.022

Bonner, J.L., Herrin, E.T., 1999, Surface wave studies of the Sierra Madre Occidental of northern Mexico: Bulletin of the Seismological Society of America, 89(5), 1323-1337.

Bryan, S., 2007, Silicic large igneous provinces: Episodes, 30(1), 20-31. https://doi. org/10.18814/epiiugs/2007/v30i1/004

Calmus, T., Pallares, G., Maury, R.C., AguillónRobles, A., Bellon, H., Benoit, M., and Michaud, F., 2010, Volcanic markers of the post-subduction evolution of Baja California and Sonora, Mexico: Slab tearing versus lithospheric rupture of the Gulf of California: Pure and Applied Geophysics, 168(8-9), 1303-1330. http://doi.org/10.1007/ s00024-010-0204-z
Cameron, K.L., Robinson, J.V., 1990, Nd-Sr isotopic compositions of lower crustal xenoliths - evidence for the origin of midTertiary felsic volcanics in Mexico: Comment: Contributions to Mineralogy and Petrology, 104, 609-618. http://doi.org/10.1007/ BF00306668

Campos-Enríquez, J.O., Kerdan, T., MoránZenteno, D.J., Urrutia-Fucugauchi, J., Sánchez-Castellanos, E., Alday-Cruz, R., 1994, Estructura de la litósfera superior a lo largo del Trópico de Cáncer: GEOS, 12, 75-76.

Castiñeiras, P., Iriondo, A., Wooden, J.L., DórameNavarro, M., Premo, W.R., 2004, Detrital zircon U-Pb SHRIMP geochronology of Proterozoic quartzites from the Bamuri Complex, NW Sonora, Mexico-evidence for a Mojave connection, en IV Reunión Nacional de Ciencias de la Tierra, Juriquilla, Querétaro, Libro de Resúmenes, 215 p.

Chen, W., Tenzer, R., 2017, Moho modeling in spatial domain: A case study under Tibet: Advances in Space Research, 59(12), 2855-2869. https://doi.org/10.1016/j. asr.2017.03.015

Coney, P.J., 1980, Cordilleran metamorphic core complexes: An overview, in Crittenden Jr., M.D., Coney, P.J., Davis, G.H. (ed.), Cordilleran Metamorphic Core Complexes, Geological Society of America, Memoir 153, 7-311. https://doi.org/10.1130/ mem 153-p7

Coney, P.J., Harms, T.A., 1984, Cordilleran metamorphic core complexes: Cenozoic extensional relics of Mesozoic compression: Geology, 12(9), 550-554. https://doi. org/10.1130/0091-7613(1984)12<550:cmc cсе>2.0.co;2

Couch, R.W, Ness, G.E., Sanchez-Zamora, O., Calderon-Riveroll, G., Doguin, P., Plawman, T., Coperude, S., Huehn, B., Gumma, W., 1991, Gravity anomalies and crustal structure of the Gulf and Peninsular Province of the Californias, in Dauphin, J.P., 
and Simoneit, B.R.T., eds., The Gulf and the Peninsular Province of the Californias: American Association of Petroleum Geologists, Memoir, 47, 47-70. https:// doi.org/10.1306/99a4d08b-3318-11d78649000102c1865d

Dixon, T., Decaix, J., Farina, F., Furlong, K., Malservisi, R., Bennett, R., Suárez-Vidal, F., Fletcher, J. Lee, J., 2002, Seismic cycle and rheological effects on estimation of presentday slip rates for the Agua Blanca and San Miguel-Vallecitos faults, northern Baja California, Mexico: Journal of Geophysical Research, Solid Earth, 107(B10), ETG-5. http://doi.org/10.1029/2000jb000099

Duque-Trujillo, J., Ferrari, L., Orozco-Esquivel, T., López-Martínez, M., Lonsdale, P., Bryan, S.E., Kluesner, J., Piñero-Lajas, D., Solari, L., 2015, Timing of rifting in the southern Gulf of California and its conjugate margins: Insights from the plutonic record: Geological Society of America Bulletin, 127(5-6), $702-736$. https://doi.org/10.1130/ b31008.1

Ebadi, S., Barzaghi, R., Safari, A., Bahroudi, A., 2019, Evaluation of different gravimetric methods to Moho recovery in Iran: Annals of Geophysics, 62(5), GD561. https://doi. org/10.4401/ag-8054

Espíndola, V.H., Quintanar, L., Espíndola, J.M., 2017, Crustal structure beneath Mexico from receiver functions: Bulletin of the Seismological Society of America, 107(5), 2427-2442. http://doi. org/10.1785/0120160152

Farmer, G.L., Bowring, S.A., Matzel, J., EspinosaMaldonado, G., Fedo, C., Wooden, J., 2005, Paleoproterozoic Mojave province in northwestern Mexico? Isotopic and U-Pb zircon geochronologic studies of Precambrian and Cambrian crystalline and sedimentary rocks, Caborca, Sonora: in Anderson, T.H., Nourse, J.A., McKee, J.W. y Steiner, M.B., eds.: The Mojave-Sonora megashear hypothesis - development, assessment, and alternatives: Geological Society of America Special Paper 393, 183-198. https://doi. org/10.1130/0-8137-2393-0.183

Fernández, A., Pérez-Campos, X., 2017, Lithosphere thickness in the Gulf of California region: Tectonophysics, 719, 17-26. http:// doi.org/10.1016/j.tecto.2017.06.016

Ferrari, L., López-Martínez, M., Rosas-Elguera, J., 2002, Ignimbrite flare-up and deformation in the southern Sierra Madre Occidental, western Mexico: Implications for the late subduction history of the Farallon plate: Tectonics, 21(4), 17-1-17-24. http://doi. org/10.1029/2001tc001302

Ferrari, L., Valencia-Moreno, M., Bryan, S., 2007, Magmatism and tectonics of the Sierra Madre Occidental and its relation with the evolution of the western margin of North America: Geological Society of America Special Paper, 422, 1-39. http://doi. org/10.1130/2007.2422(01)

Ferrari, L., López-Martínez, M., Orozco-Esquivel, T., Bryan, S.E., Duque-Trujillo, J., Lonsdale, P. Solari, L., 2013, Late Oligocene to Middle Miocene rifting and synextensional magmatism in the southwestern Sierra Madre Occidental, Mexico: The beginning of the Gulf of California rift: Geosphere, 9(5), 1161-1200. http://doi.org/10.1130/ ges00925.1

Fletcher, J.M., Kohn, B.P., Foster, D.A., Gleadow, A.J., 2000, Heterogeneous Neogene cooling and exhumation of the Los Cabos block, southern Baja California: Evidence from fission-track thermochronology: Geology, 28(2), 107-110. http://doi. org/10.1130/0091-7613(2000)28<107:HN CAEO $>2.0 . \mathrm{CO} ; 2$

Fragoso-Irineo, A., 2014, Análisis de datos aeromagnéticos y gravimétricos del sector septentrional del Arco Alisitos: implicaciones tectónicas: Centro de Investigación Científica y de Educación Superior de Ensenada, Baja California, Tesis de maestría en Ciencias, 118 p. 
Gaite, B., Villaseñor, A., Iglesias, A., Herraiz, M., Jiménez-Munt, I., 2015, A 3-D shear velocity model of the southern North America and the Caribbean plates from ambient noise and earthquake tomography: Solid Earth Discussions, 6, 2971-3002. http://doi. org/10.5194/sed-6-2971-2014

García-Abdeslem, J., 2017, Nonlinear inversion of isostatic residual gravity data from Montage Basin, northern Gulf of California: Geophysics, 82, 3, G45-G55. http://doi.org/10.1190/ geo2016-0144.1

Gastil, R.G., Phillips, R.P., Allison, E.C., 1975, Reconnaissance geology of the state of Baja California: Geological Society of America, Memoir 140, 170 p. https://doi.org/10.1130/ mem 140-p 1

Geosoft, 2015, Oasis Montaj 8.4, Geosoft Inc., Toronto, Canada. http://www.geosoft.com

Gómez-Ortiz, D., Agarwal, B.N., 2005, 3DINVER.m: a MATLAB program to invert the gravity anomaly over a 3D horizontal density interface by Parker-Oldenburg's algorithm: Computers and Geosciences, 31(4), 513-520. http://doi.org/10.1016/j. cageo.2004.11.004

González-Fernández, A., Dañobeitia, J.J., DelgadoArgote, L.A., Michaud, F., Córdoba, D., Bartolomé, R., 2005, Mode of extension and riftinghistory of upperTiburón andupperDelfín basins, northern Gulf of California: Journal of Geophysical Research, Solid Earth, 110, B1. http://doi.org/10.1029/2003jb002941

Gruber, T., Rummel, R., 2013, The $4^{\text {th }}$ release of GOCE gravity field models-overview and performance analysis, in EGU general assembly, Vienna, Austria, 15, 1357.

Hayford, J.F., Bowie, W.,1912, Geodesy: the effect of topography and isostatic compensation upon the intensity of gravity, US Government Printing Office, 4.

Hendricks, J.D., Plescia, J.B., 1991, A review of the regional geophysics of the Arizona transition zone: Journal of Geophysical Research, Solid Earth, 96(B7), 12351-12373. http://doi. org/10.1029/90jb01781
Henry, C.D., Aranda-Gómez, J.J., 2000, Plate interactions control middle-late Miocene, proto-Gulf and Basin and Range extension in the southern Basin and Range: Tectonophysics, 318(1), 1-26. https://doi. org/10.1016/s0040-1951(99)00304-2

Ichinose, G., Day, S., Magistrale, H., Prush, T., Vernon, F., Edelman, A., 1996, Crustal thickness variations beneath the peninsular ranges, southern California: Geophysical Research Letters, 23(22), 3095-3098. http:// doi.org/10.1029/96gl03020

Ince, E.S., Barthelmes, F., Reißland, S., Elger, K., Förste, C., Flechtner, F., Schuh, H., 2019, ICGEM - 15 years of successful collection and distribution of global gravitational models, associated services and future plans: Earth System Science Data, 11(2), 647-674. http://doi.org/10.5194/essd-11-647-2019

Iriondo, A., Premo, W.R., 2011, Las rocas cristalinas proterozoicas de Sonora y su importancia para la reconstrucción del margen continental SW de Laurencia La pieza mexicana del rompecabezas de Rodinia, in Calmus, T. (ed.), Panorama de la geología de Sonora, México: Universidad Nacional Autónoma de México, Instituto de Geología, Boletín 118, 25-55.

Kane, M.F., 1962, A comprehensive system of terrain corrections using a digital computer. Geophysics, 27(4), 455-462. http://doi. org/10.1190/1.1439044

Karig, D.E., Jensky, W., 1972, The proto-gulf of California: Earth and Planetary Science Letters, 17(1), 169-174. https://doi. org/10.1016/0012-821x(72)90272-5

Kerdan, T.P., 1992, Estructura de la corteza y manto superior en el norte de México (a lo largo del Trópico de Cáncer desde Baja California hasta el Golfo de México): México, Universidad Nacional Autónoma de México, Colegio de Ciencias y Humanidades, Unidad Académica de los Ciclos Profesionales y de Posgrado, Tesis de maestría, $347 \mathrm{p}$.

Langenheim, V.E., Jachens, R.C., Aiken, C., 2014, Geophysical framework of the 
Peninsular Ranges batholith: Implications for tectonic evolution and neo- tectonics: in Morton, D.M., Miller, F.K. (eds.), Peninsular Ranges Batholith, Baja California and Southern California: Geological Society of America Memoir 211, 1-20. https://doi. org/10.1130/2014.1211(01)

Laske, G., Masters, G., Ma, Z., Pasyanos, M., 2013, Update on CRUST1. 0-A 1-degree global model of Earth's crust (abstract), in EGU General Assembly, Vienna, Austria, 15, 2658.

Lazcano-Prieto, J., 2018, Evolución tectónica del noroeste de México y su relación con los yacimientos de oro orogénico: Universidad Nacional Autónoma de México, Tesis de maestría, $125 \mathrm{p}$.

Lee, D.H., Acharya, T.D., 2017, Comparison of complete bouguer anomalies from satellite marine gravity models with shipborne gravity data in East Sea: Journal of Marine Science and Technology, 25(6), 625-632. http://doi. org/10.6119/JMST-017-1226-01

Lewis, J.L., Day, S.M., Magistrale, H., Eakins, J., Vernon, F., 2000, Regional crustal thickness variations of the Peninsular Ranges, southern California: Geology, 28(4), 303-306. http://doi.org/10.1130/0091$7613(2000) 28<303:$ rctvot $>2.0$. co;2

Lewis, J.L., Day, S.M., Magistrale, H., Castro, R.R., Rebollar, C., Astiz, L., Eakins, J., Vernon F.L., Brune, J.N., 2001, Crustal thickness of the Peninsular Ranges and Gulf extensional province in the Californias: Journal of Geophysical Research, 106(13), 13599-13611. http://doi. org/10.1029/2001jb000178

Lizarralde, D., Axen, G.J., Brown, H.E., Fletcher, J.M., González-Fernández, A., Harding, A.J., Holbrook, W.S., Kent, G.M., Paramo, P., Sutherland, F., Umhoefer, P.J., 2007, Variation in styles of rifting in the Gulf of California: Nature, 448(7152), 466-469. http://doi.org/10.1038/nature06035

Lonsdale, P., 1989, Geology and tectonic history of the Gulf of California, in Winterer, E.L., Hussong, D.M., Decker, R.W. (eds.), The Eastern Pacific Ocean and Hawaï: Boulder, Colorado, Geological Society of America, The Geology of North America, N, 499-521. https://doi.org/10.1130/dnag-gna-n.499

López-Pineda, L., Rebollar, G.J., Quintanar, L., 2007, Crustal thickness estimates for Baja California, Sonora, and Sinaloa, Mexico, using disperse surface waves: Journal of Geophysical Research, 112, B04308. http:// doi.org/10.1029/2005JB003899

Lowrie, W., 2007, Fundamentals of Geophysics: Cambridge University Press, Cambridge, 354 pp. https://doi.org/10.1017/ cbo9780511807107

Martín-Barajas, A., M. González-Escobar, J. M. Fletcher, M. Pacheco, M. Oskin, and R. Dorsey, 2013, Thick deltaic sedimentation and detachment faulting delay the onset of continental rupture in the Northern Gulf of California: Analysis of seismic reflection profiles: Tectonics, 32(5), 1294-1311. https://doi.org/10.1002/tect.20063

Moore, D.G., 1973, Plate-edge deformation and crustal growth, Gulf of California structural province: Geological Society of America Bulletin, 84, 1883-1905. http://doi. org/10.1130/0016-7606(1973)84<1883:PD ACGG > 2.0.CO;2

Nagy, D., 1966, The gravitational attraction of a right rectangular prism: Geophysics, 31(2), 362-371. doi:10.1190/1.1439779

Nourse, J. A., Anderson, T. H., Silver, L. T., 1994, Tertiary metamorphic core complexes in Sonora, northwestern Mexico: Tectonics, 13(5), 1161-1182. http://doi. org/10.1029/93tc03324

O’Donnell, J. P., Nyblade, A. A., 2014, Antarctica's hypsometry and crustal thickness: Implications for the origin of anomalous topography in East Antarctica: Earth and Planetary Science Letters, 388, 143-155. https://doi.org/10.1016/j.epsl.2013.11.051

Oldenburg, D.W., 1974, The inversion and interpretation of gravity anomalies: 
Geophysics, 39(4), 526-536. http://doi. org/10.1190/1.1440444

Oskin, M., Stock, J., Martín-Barajas, A., 2001, Rapid localization of Pacific-North America plate motion in the Gulf of California: Geology, 29(5), 459-462. http://doi. org/10.1130/0091-7613(2001)029<0459:rl opna>2.0.co;2

Parker, R.L., 1972, The rapid calculation of potential anomalies: Geophysical Journal of the Royal Astronomical Society, 31(4), 447-455. http://doi.org/10.1111/j.1365246x. 1973.tb06513.x

Paulssen, H., de Vos, D., 2017, Slab remnants beneath the Baja California peninsula: Seismic constraints and tectonic implications: Tectonophysics, 719, 27-36. http://doi. org/10.1016/j.tecto.2016.09.021

Persaud, P., Pérez-Campos, X., Clayton, R.W., 2007, Crustal thickness variations in the margins of the Gulf of California from receiver functions: Geophysical Journal International, 170(2), 687-699. http://doi. org/10.1111/j.1365-246x.2007.03412.x

Reston, T.J., 2009, The extension discrepancy and syn-rift subsidence deficit at rifted margins: Petroleum Geoscience, 15(3), 217-237. http://doi.org/10.1144/1354-079309-845

Reyes, L.M., Rebollar, C.J., Castro, R., 2001, Depth of the Moho in northern Baja California using (Pg-Pn) travel times: Geofísica Internacional, 40(1), 21-29.

Ries, J., Bettadpur, S., Eanes, R., Kang, Z., Ko, U., McGullough, C., Nagel, P., Pie, N., Poole, S., Richter, T., Save, H., Tapley, B., 2016, The Development and Evaluation of the Global Gravity Model GGM05, CSR Report CSR16-02: Center for Space Research, The University of Texas at Austin. https://doi. org/10.26153/tsw/1461

Ryan, W.B.F., Carbotte, S.M., Coplan, J.O., O'Hara, S., Melkonian, A., Arko, R., Weissel, R.A., Ferrini, V., Goodwillie, A., Nitsche, F., Bonczkowski, J., Zemsky, R., 2009, Global Multi-Resolution Topography synthesis: Geochemistry, Geophysics,
Geosystems, 10(3), Q03014. http://doi. org/10.1029/2008GC002332

Sánchez-Zamora, O., 1981, Gravity and structure of the Pacific continental margin of central Mexico: Oregon State University, tesis de maestría, $39 \mathrm{p}$.

Schaaf, P., Böhnel, H. Pérez-Venzor, J.A., 2000, Pre-Miocene palaeogeography of the Los Cabos block, Baja California sur: Geochronological and palaeomagnetic constraints: Tectonophysics, 318(1-4), 53-69. http://doi.org/10.1016/ s0040-1951(99)00306-6

Schaaf, P., Díaz-López, F., Gutiérrez-Aguilar, F., Solís-Pichardo, G., Hernández-Treviño, T., Arrieta-García, G., Solari, L., OrtegaObregón, C., 2019, Geochronology and geochemistry of the Puerto Vallarta igneous and metamorphic complex and its relation to Cordilleran arc magmatism in northwestern Mexico: Lithos, 105248. https://doi. org/10.1016/j.lithos.2019.105248

Sheth,H.C., 2007, 'LargeIgneousProvinces(LIPs)': Definition, recommended terminology, and a hierarchical classification. Earth-Science Reviews, 85(3-4), 117-124. http://doi. org/10.1016/j.earscirev.2007.07.005

Spencer, J., 2018, Southwest North America corecomplex belt, in The University of Arizona College of Science, The Arizona Geological Survey. <https://azgs.arizona.edu/photo/ southwest-north-america-core-complexbelt>, consulta 4 de mayo de 2018.

Stock, J.M., Hodges, K.V., 1989, Pre-Pliocene extension around the Gulf of California and the transfer of Baja California to the Pacific plate: Tectonics, 8(1), 99-115. http://doi. org/10.1029/TC008i001p00099

Sylvester, A.G., Smith, R.R., 1976, Tectonic transpression and basement-controlled deformation in San Andreas fault zone, Salton Trough, California: AAPG Bulletin, 60(12), 2081-2102. https://doi.org/10.1306/ clea3a73-16c9-11d7-8645000102c1865d

Thatcher, W., Brune, J.N., 1973, Surface waves and crustal structure in the Gulf of California 
region: Bulletin of the Seismological Society of America, 63(5), 1689-1698.

Tugume, F., Nyblade, A., Julià, J., van der Meijde, M., 2013, Precambrian crustal structure in Africa and Arabia: Evidence lacking for secular variation: Tectonophysics, 609, 250-266. http://doi.org/10.1016/j. tecto.2013.04.027

Umhoefer, P.J., Dorsey, R.J., Willsey, S., Mayer, L., Renne, P., 2001, Stratigraphy and geochronology of the Comondu Group near Loreto, Baja California Sur, Mexico: Sedimentary Geology, 144(1-2), 125-147. http://doi.org/10.1016/ s0037-0738(01)00138-5

Van der Meijde, M., Julià, J., Assumpção, M., 2013, Gravity derived Moho for South America: Tectonophysics, 609, 456-467. http://doi.org/10.1016/j.tecto.2013.03.023

Vega-Granillo, R., Calmus, T., 2003, Mazatan metamorphic core complex (Sonora, Mexico): structures along the detachment fault and its exhumation evolution: Journal of South American Earth Sciences, 16(4), 193-204. http://doi.org/10.1016/ s0895-981 1(03)00066-x

Wang, Y., Forsyth, D.W., Savage, B., 2009, Convective upwelling in the mantle beneath the Gulf of California: Nature 462(7272), 499-501. http://dx.doi.org/10.1038/ nature 08552

Wetmore, P.H., Herzig, C., Alsleben, H., Sutherland, M., Schmidt, K.L., Schultz, P.W., Paterson, S.R., 2003, Mesozoic tectonic evolution of the Peninsular Ranges of southern and Baja California, in Johnson, S.E., Paterson, S.R., Fletcher, J.M., Girty, G.H., Kimbrough, D.L., Martín-Barajas, A. (eds.), Tectonic Evolution of Western Mexico and the Southwestern USA: Boulder, Colorado, Geological Society of America
Special Paper, 374, 93-116.

Wetmore, P.H., Schmidt, K.L., Paterson, S.R. Herzig, C., 2002, Tectonic implications for the along-strike variation of the Peninsular Ranges batholith, southern and Baja California: Geology, 30(3), 247-250. http://doi. org/10.1130/0091-7613(2002)030<0247:tif tas>2.0.co;2

Whitman, W.W., 1991, A microgal approximation for the Bullard B - earth's curvature - gravity correction: Geophysics, 56(12), 1980-1985. http://doi.org/10.1190/1.1443009

Wong, M.S., Gans, P.B., 2003, Tectonicimplications of early Miocene extensional unroofing of the Sierra Mazatán metamorphic core complex, Sonora, Mexico: Geology, 31(11), 953-956. http://doi.org/10.1130/g19843.1

Wong, M.S., Gans, P.B., 2008, Geologic,structural and thermochronological constraints on the tectonic evolution of the Sierra Mazatán core complex, Sonora, Mexico: New insighits into metamorphic core complex formation: Tectonics 27(TC4013). http:// doi.org/10.1029/2007TC002173

Wong, M.S., Gans, P.B., Scheier, J., 2010, The $40 \mathrm{Ar} / 39 \mathrm{Ar}$ thermochronology of core complexesandotherbasementrocksinSonora, Mexico: Implications for Cenozoic tectonic evolution of northwestern Mexico: Journal of Geophysical Research, 115, B07414. http://doi.org/10.1029/2009JB007032

Zhao, G., Liu, J., Chen, B., Kaban, M. K., Zheng, X., 2020, Moho beneath Tibet based on a joint analysis of gravity and seismic data: Geochemistry, Geophysics, Geosystems, 21(2). http://doi.org/10.1029/2019gc008849

Zhu,L., Kanamori,H., 2000, Mohodepth variation in southern California from teleseismic receiver functions: Journal of Geophysical Research: Solid Earth, 105, B2, 2969-2980. http://doi.org/10.1029/1999jb900322 